\title{
Genetic variances, trends and mode of inheritance for hip and elbow dysplasia in Finnish dog populations
}

\author{
K. Mäki ${ }^{1+}$, A. F. Groen ${ }^{2}$, A.-E. Liinamo ${ }^{3}$ and M. Ojala ${ }^{1}$ \\ ${ }^{1}$ Department of Animal Science, PO Box 28, 00014 Helsinki University, Finland \\ ${ }^{2}$ Animal Breeding and Genetics Group, Wageningen University, PO Box 338, 6700 AH Wageningen, The Netherlands \\ ${ }^{3}$ Farm Animal Industrial Platform, Benedendorpsweg 98, 6862 WL Oosterbeek, The Netherlands
}

†E-mail: katariina.maki@animal.helsinki.fi

\begin{abstract}
The aims of this study were to assess genetic variances, trends and mode of inheritance for hip and elbow dysplasia in Finnish dog populations. The influence of time-dependent fixed effects in the model when estimating the genetic trends was also studied. Official hip and elbow dysplasia screening records of 42421 dogs from seven breeds were analysed with REML. To investigate the mode of inheritance of hip and elbow dysplasia, trait distributions, genetic variances and regressions of offspring phenotypes on parental predicted breeding values were studied separately in males and in females. Genetic trends for hip dysplasia between the years 1983 and 1998 were favourable only in the Rottweiler. In elbow dysplasia, the trends were favourable after the year 1992 in all the four breeds studied but the overall changes were small. The reason for this seemed to be negligible selection pressure against these traits. Timedependent fixed effects in the model had an influence on the estimated genetic trends, resulting either in a more negative or more positive genetic trend compared with the model from which the time-dependent effects were removed. Mitochondrial or sex-linked inheritance did not seem likely in the expression of hip and elbow dysplasia in the populations studied. Regression coefficients of offspring phenotypes on estimated parental breeding values were approximately equal to their expected value in a situation with equal parental contribution. Furthermore, the phenotypic frequency distributions of hip and elbow dysplasia grades were similar among males and females in each breed studied. No indication of major genes was found in the offspring frequency distributions within individual sires. According to these Finnish data, mode of inheritance for both hip and elbow dysplasia is polygenic (quantitative) with equal expression of the genes from both parents, although the estimates of heritability for hip dysplasia in the Rough Collie and for elbow dysplasia in the German Shepherd and the Golden Retriever were somewhat different in males compared with females.
\end{abstract}

Keywords: dogs, dysplasia, genetic parameters, inheritance.

\section{Introduction}

Hip and elbow dysplasia are the most common heritable diseases among large-sized dogs. They are growth disorders of the bone and considered as highly disabling. Attempts to reduce the incidence of canine hip and elbow dysplasia in Finland like in many other countries have been based mainly on radiographic examination of the joints and consequent phenotypic selection, with a varying selection intensity between the breeds and the countries. The Finnish Kennel Club's (FKC) control programme for hip dysplasia started in 1984 for the
Golden and Labrador Retriever, and more breeds and defects have been introduced in it during the years. The programme is basically the same for all breeds involved, with only minor differences in required threshold values for breeding animals.

Moderate to high heritabilities $\left(h^{2}\right)$ have been reported for both hip (e.g. Leighton et al., 1977; Hedhammar et al., 1979; Leppänen et al., 2000) and elbow (Guthrie and Pidduck, 1990; Grøndalen and Lingaas, 1991; Mäki et al., 2000) dysplasia, indicating that some genetic progress could result even from 
phenotypic selection. Noticeable phenotypic progress has been achieved mostly in closed breeding populations with systematic breeding programmes (Hedhammar et al., 1979; Leighton, 1997; Ohlerth et al., 2001), although some gain has occurred in other populations as well (Brass, 1989; Swenson et al., 1997a and b; Beuing et al., 2000; Reed et al., 2000). In Finland, no phenotypic gain in hip dysplasia has been observed in most breeds (Leppänen and Saloniemi, 1999). One reason for this could be that selection against hip dysplasia has not been very effective, since other selection criteria, like success in the shows, are often considered more important than the health of the joints. Other reasons could be the lack of accurately estimated breeding values for these traits, or a selection strategy that is not optimized for the (true) mode of inheritance.

Hip and elbow dysplasia have been shown to be at least partly quantitative, i.e., polygenic traits (e.g. Leighton et al., 1977; Brass, 1989; Swenson et al., 1997a). Polygenes involved can have additive, dominance and epistatic effects. In addition to polygenes, a single gene with a large effect, i.e. a major gene, could be segregating. Moreover, the mode of inheritance could be maternal (mitochondrial DNA) or sex-linked (Xchromosomal). In a few studies, the pattern of inheritance for fragmented coronoid process (FCP), which is one of the four possible growth disorders in elbow dysplasia, has been found to be complex; it has been suggested to be controlled by imprinted or sex-linked genes (Janss and Brascamp, 1998) or by a major gene (Everts, 2000). Janss and Brascamp (1998) found a high heritability for FCP, but the regression of offspring phenotypes on the pedigree index (the mean of the estimated breeding values of the sire and the dam) was -0.25 instead of the expected value $1 \cdot 0$. However, the data set studied was very small. Some evidence is provided for genetic variances and/or penetrance of the genes being different for both sexes (Everts, 2000; Wood et al., 2000a).

The aims of this study were to estimate variance components and to assess genetic trends in hip and elbow dysplasia during the years 1983 to 1998. The influence of time-dependent fixed effects in the model when estimating the genetic trends, and the mode of inheritance of hip and elbow dysplasia in Finnish dog populations were also studied.

\section{Material and methods}

\section{Material}

Data and pedigree information used in this study were obtained from the FKC. Seven breeds were chosen according to the highest number of dysplasiascreened dogs: German Shepherd (GS), Golden
Retriever (GR), Labrador Retriever (LR), Rough Collie (CO), Rottweiler (RO), Bernese Mountain Dog (BM) and Finnish Hound (FH) (Tables 1 and 2). The $\mathrm{CO}$ and the $\mathrm{FH}$ had only a few observations on elbow dysplasia and were therefore omitted from the analyses concerning that trait. The screening data consisted of the official hip and elbow dysplasia screening records of the dogs screened during the years 1988 to 2000 . Included were the identification number of the dog, date of screening, identification numbers of both the X-raying and the screening veterinarian (radiologist) and a grade for both left and right hip and/or elbow joint.

Hip dysplasia grading had been done with three different systems during the time the data covered. The different grading systems were combined as in a previous study on hip and elbow dysplasia (Mäki et al., 2001), to eight numerical values: 0 and 0.5 (normal), 1 (borderline), 2 (mild dysplasia), 3 (moderate dysplasia), and $4 \cdot 0,4.5$, and 5.0 (severe dysplasia). A diagnosis of hip dysplasia requires malformation of the joint, subluxation or a perfect luxation. Elbow joints were graded according to International Elbow Working Group protocol, with numbers from 0 to 3 . The elbow score 0 represents dogs having normal or borderline elbow joints and the scores 1, 2 and 3 dogs with mild, moderate and severe elbow dysplasia, respectively. Elbows are classified according to the grade of arthrosis in the elbow joint. Four possible growth disorders may result on the same phenotype : elbow incongruity (INC), fragmented coronoid process (FCP), osteochondritis dissecans (OCD), and ununited anconeal process (UAP). Hip and elbow grades were not normally distributed because most dogs were graded as healthy or borderline (Mäki et al., 2001).

For both hip and elbow dysplasia, the measures used in statistical analyses were the means of the screening results for left and for right joint of each dog with 1.0 added for the statistical programs, which otherwise would have treated the phenotypic value zero as a missing observation.

Pedigree information used in the analyses was obtained from the register of the seven breeds, kept by the FKC. The database included the identification number of the dog and its parents, sex code, breed, breeder of the dog, and date of birth. Litter identification was established assuming that all dogs that were born on the same date from the same parents were littermates. Litter size was calculated only for dogs born in Finland because imported dogs did not have all littermates in the Finnish registry. Screening age of the dog and age of the dam at the 
Table 1 Estimates of additive genetic variance $\left(\sigma_{\mathrm{a}}^{2}\right)$ and variance ratios (relative to $\sigma_{\mathrm{p}}^{2}$ ) for hip dysplasia; separately for data sets including both sexes $(a)$, only females $(f)$ and only males $(m)$

\begin{tabular}{|c|c|c|c|c|c|c|c|c|}
\hline \multirow{2}{*}{\multicolumn{2}{|c|}{$\begin{array}{l}\text { Source of } \\
\text { variation }\end{array}$}} & \multicolumn{7}{|c|}{ Breedt } \\
\hline & & GS & GR & LR & $\mathrm{CO}$ & $\mathrm{RO}$ & $\mathrm{BM}$ & $\mathrm{FH}$ \\
\hline \multirow{3}{*}{\multicolumn{2}{|c|}{$\begin{array}{l}\text { No. } \\
\text { Females (\%) } \\
\text { Nped.f }\end{array}$}} & 13006 & 8440 & 7826 & 4337 & 4397 & 2271 & 2144 \\
\hline & & 56 & 57 & 56 & 63 & 54 & 58 & 49 \\
\hline & & 22095 & 11674 & 11864 & 7602 & 6471 & 3551 & 9437 \\
\hline \multirow{3}{*}{$\sigma^{2} a$} & a & 0.26 & 0.33 & 0.31 & $0 \cdot 15$ & $0 \cdot 46$ & 0.49 & 0.37 \\
\hline & $\mathrm{f}$ & $0 \cdot 21$ & $0 \cdot 26$ & $0 \cdot 25$ & $0 \cdot 11$ & $0 \cdot 37$ & $0 \cdot 42$ & 0.29 \\
\hline & $\mathrm{m}$ & $0 \cdot 20$ & $0 \cdot 44$ & $0 \cdot 25$ & 0.21 & 0.36 & 0.57 & 0.36 \\
\hline \multirow[t]{3}{*}{ Animal $\left(h^{2}\right)$} & a & $0 \cdot 24 \pm 0 \cdot 02$ & 0.29 & $0 \cdot 26 \pm 0 \cdot 02$ & $0 \cdot 20 \pm 0 \cdot 03$ & $0 \cdot 38 \pm 0 \cdot 01$ & $0 \cdot 37 \pm 0 \cdot 02$ & $0 \cdot 41 \pm 0 \cdot 03$ \\
\hline & $\mathrm{f}$ & $0 \cdot 18 \pm 0 \cdot 02$ & $0 \cdot 19 \pm 0 \cdot 02$ & $0 \cdot 21 \pm 0 \cdot 02$ & $0 \cdot 13 \pm 0 \cdot 03$ & $0 \cdot 31 \pm 0 \cdot 02$ & $0 \cdot 32 \pm 0.04$ & $0 \cdot 32 \pm 0 \cdot 05$ \\
\hline & $\mathrm{m}$ & $0 \cdot 19 \pm 0 \cdot 02$ & $0 \cdot 31 \pm 0 \cdot 04$ & $0.21 \pm 0.03$ & $0 \cdot 29 \pm 0 \cdot 05$ & $0 \cdot 31 \pm 0 \cdot 05$ & 0.42 & $0 \cdot 38 \pm 0 \cdot 05$ \\
\hline \multirow[t]{3}{*}{ Litter } & a & $0 \cdot 06 \pm 0 \cdot 01$ & 0.05 & $0 \cdot 01 \pm 0 \cdot 01$ & $0 \cdot 06 \pm 0 \cdot 01$ & $0 \cdot 04 \pm 0 \cdot 01$ & $0 \cdot 04 \pm 0 \cdot 01$ & $0.02 \pm 0.03$ \\
\hline & $\mathrm{f}$ & $0 \cdot 06 \pm 0 \cdot 01$ & $0 \cdot 06 \pm 0 \cdot 01$ & $0 \cdot 02 \pm 0 \cdot 01$ & $0 \cdot 06 \pm 0 \cdot 02$ & $0.06 \pm 0.02$ & $0.05 \pm 0.02$ & $0.09 \pm 0.05$ \\
\hline & $\mathrm{m}$ & $0 \cdot 10 \pm 0 \cdot 01$ & $0 \cdot 06 \pm 0 \cdot 02$ & $0 \cdot 01 \pm 0 \cdot 01$ & $0 \cdot 06 \pm 0 \cdot 03$ & $0 \cdot 10 \pm 0 \cdot 02$ & 0.04 & $0.02 \pm 0.04$ \\
\hline \multirow[t]{3}{*}{ Breeder } & $\mathrm{a}$ & $0.06 \pm 0.01$ & 0.05 & $0 \cdot 07 \pm 0 \cdot 01$ & $0 \cdot 08 \pm 0 \cdot 01$ & $0 \cdot 05 \pm 0 \cdot 01$ & $0 \cdot 04 \pm 0.01$ & $0 \cdot 02 \pm 0 \cdot 01$ \\
\hline & $\mathrm{f}$ & $0 \cdot 12 \pm 0 \cdot 01$ & $0 \cdot 09 \pm 0 \cdot 01$ & $0 \cdot 14 \pm 0 \cdot 02$ & $0 \cdot 14 \pm 0 \cdot 02$ & $0 \cdot 05 \pm 0 \cdot 01$ & $0.06 \pm 0.02$ & $0 \cdot 12 \pm 0 \cdot 04$ \\
\hline & $\mathrm{m}$ & $0 \cdot 04 \pm 0 \cdot 01$ & $0 \cdot 04 \pm 0 \cdot 01$ & $0 \cdot 05 \pm 0 \cdot 01$ & $0.07 \pm 0.02$ & $0 \cdot 06 \pm 0.01$ & 0.06 & $0 \cdot 01 \pm 0 \cdot 01$ \\
\hline
\end{tabular}

+ GS = German Shepherd, GR = Golden Retriever, LR = Labrador Retriever, CO= Rough Collie, RO= Rottweiler, $\mathrm{BM}=$ Bernese Mountain Dog, $\mathrm{FH}=$ Finnish Hound.

No standard errors were obtained for data set ' $a$ ' in GR and data set ' $m$ ' in BM.

$\ddagger$ Nped. = number of animals in the relationship matrix.

time the litter was born were added to the data, after first merging the pedigree information with the screening data.

\section{Methods \\ Statistical model for estimating variance components and breeding values. A statistical model for hip and elbow dysplasia was fitted first for the data of each breed specifically, and then one general model, including the effects that were statistically significant in more than one breed, was chosen for the estimation of variance components in all breeds. The fixed and random effects tested in the models were the sex of dog, year of birth, season of birth, year of birth $X$ season of birth subclass, age of dog at screening, breeder, litter size, age of dam at the time the litter was born, veterinarian doing the X-ray, radiologist responsible for grading each radiograph, and maternal (effects common to the offspring of the same dam) and litter effects. Breeder and veterinarian doing the $\mathrm{X}$-ray as well as maternal and litter effects were considered as random, while the others were considered fixed.}

When estimating variance components and breeding values, the following model for both hip and elbow dysplasia was used:

$$
\begin{aligned}
& y_{i j k l m n o p}=\mu+a g e_{i}+\text { sex }_{j}+\text { year }_{k}+\text { rad }_{l}+\text { vet }_{m}+\text { breeder }_{n} \\
& + \text { lit }_{o}+a_{p}+\varepsilon_{i j k l m \text { по }} \text {, }
\end{aligned}
$$

where $y_{i j k l m \text { по }}=$ a screening result for hip or elbow dysplasia, $\mu=$ overall mean, $a g e_{i}=$ fixed effect of the $i$ th age class ( $i=1$ to 10,11 or 12 , depending on the trait and the breed), sex $x_{j}=$ fixed effect of the $j$ th sex, year $_{k}=$ fixed effect of the $k$ th year of birth $(k=1$ to 8 , $9,10,11$ or 13 , depending on the trait and the breed), $\mathrm{rad}_{l}=$ fixed effect of the $l$ th radiologist $(l=1$ to 5$)$, vet $_{m}=$ random effect of the $m$ th X-raying veterinarian, breeder $_{n}=$ random effect of the $n$th dog breeder, $\quad l i t_{o}=$ random effect of the oth litter, $a_{p}=$ random additive genetic effect of the $p$ th animal, and $\varepsilon_{i j k l m n o p}=$ random residual effect.

Age was classified in 10 to 12 classes, depending on the breed, from twelve months upwards. Radiologist was an effect of the five radiologists grading the Xrays, and was included in the FKC's data only from the beginning of the year 1994 onward. Since December 1994, there has been only one radiologist at any one time : one until January 1996 and another since that. Altogether 179 to 421 veterinarians, 285 to 1431 breeders and 792 to 4791 litters existed in the data, depending on the breed. The distributions of vet, breeder, lit, $a$ and $\varepsilon$ were assumed to be multivariate normal with zero means and with $\operatorname{Var}($ vet $)=\mathrm{I} \sigma_{\text {vet }}^{2}, \operatorname{Var}($ breeder $)=\mathrm{I} \sigma_{\text {breeder }}^{2} \operatorname{Var}($ lit $)=\mathrm{I} \sigma_{\text {lit }}^{2}$, 
Table 2 Estimates of additive genetic variance $\left(\sigma^{2}\right.$ ) and variance ratios (relative to $\sigma^{2} \mathrm{p}$ ) for elbow dysplasia; separately for data sets including both sexes (a), only females $(f)$ and only males $(m)$

\begin{tabular}{|c|c|c|c|c|c|c|}
\hline \multirow{2}{*}{\multicolumn{2}{|c|}{ Source of variation }} & \multicolumn{5}{|c|}{ Breedt } \\
\hline & & GS & GR & LR & $\mathrm{RO}$ & $\mathrm{BM}$ \\
\hline & 3121 & 2640 & 2627 & 3386 & 1769 \\
\hline \multicolumn{2}{|l|}{ Females (\%) } & 57 & 55 & 55 & 54 & 59 \\
\hline \multicolumn{2}{|l|}{ Nped.f } & 22095 & 11674 & 11864 & 6471 & 3551 \\
\hline \multirow{3}{*}{$\sigma_{a}^{2}$} & $\mathrm{a}$ & 0.04 & 0.08 & 0.03 & $0 \cdot 17$ & $0 \cdot 11$ \\
\hline & $\mathrm{f}$ & $0 \cdot 01$ & $0 \cdot 10$ & $0 \cdot 02$ & $0 \cdot 15$ & $0 \cdot 12$ \\
\hline & $\mathrm{m}$ & 0.05 & 0.08 & 0.08 & 0.19 & $0 \cdot 17$ \\
\hline \multirow[t]{3}{*}{ Animal $\left(h^{2}\right)$} & a & $0 \cdot 15 \pm 0 \cdot 02$ & $0 \cdot 26$ & $0 \cdot 10 \pm 0 \cdot 03$ & $0.37 \pm 0.03$ & $0 \cdot 17 \pm 0 \cdot 02$ \\
\hline & $\mathrm{f}$ & $0 \cdot 05 \pm 0 \cdot 03$ & $0 \cdot 27 \pm 0 \cdot 03$ & $0 \cdot 10 \pm 0 \cdot 02$ & $0 \cdot 38 \pm 0 \cdot 04$ & $0 \cdot 20 \pm 0 \cdot 04$ \\
\hline & $\mathrm{m}$ & $0 \cdot 15 \pm 0 \cdot 02$ & $0 \cdot 15 \pm 0 \cdot 05$ & $0 \cdot 19 \pm 0 \cdot 08$ & $0 \cdot 33 \pm 0 \cdot 03$ & 0.22 \\
\hline \multirow[t]{3}{*}{ Litter } & $\mathrm{a}$ & $0 \cdot 08 \pm 0.02$ & 0.07 & $0 \cdot 11 \pm 0 \cdot 02$ & $0 \cdot 04 \pm 0 \cdot 01$ & $0 \cdot 11 \pm 0 \cdot 02$ \\
\hline & $\mathrm{f}$ & $0 \cdot 36 \pm 0 \cdot 04$ & $0 \cdot 11 \pm 0 \cdot 03$ & $0 \cdot 10 \pm 0 \cdot 03$ & $0.02 \pm 0.02$ & $0.05 \pm 0.02$ \\
\hline & $\mathrm{m}$ & $0 \cdot 04 \pm 0 \cdot 02$ & $0 \cdot 18 \pm 0 \cdot 04$ & $0 \cdot 20 \pm 0 \cdot 04$ & $0 \cdot 07 \pm 0.02$ & 0.36 \\
\hline \multirow[t]{3}{*}{ Breeder } & $\mathrm{a}$ & $0 \cdot 09 \pm 0.01$ & 0.01 & $0 \cdot 05 \pm 0 \cdot 01$ & $0 \cdot 01 \pm 0 \cdot 00$ & $0 \cdot 05 \pm 0 \cdot 01$ \\
\hline & $\mathrm{f}$ & $0.04 \pm 0.03$ & $0 \cdot 01 \pm 0 \cdot 01$ & $0 \cdot 14 \pm 0 \cdot 02$ & $0 \cdot 01 \pm 0 \cdot 00$ & $0.07 \pm 0.02$ \\
\hline & $\mathrm{m}$ & $0 \cdot 10 \pm 0 \cdot 02$ & $0 \cdot 01 \pm 0 \cdot 01$ & $0.02 \pm 0.04$ & $0 \cdot 03 \pm 0 \cdot 01$ & 0.01 \\
\hline
\end{tabular}

话 See Table 1 footnotes.

$\operatorname{Var}(a)=\mathrm{A} \sigma_{a}^{2}$, and $\operatorname{Var}(\varepsilon)=\mathrm{I} \sigma^{2} \varepsilon$. Covariances between vet, breeder, lit, $a$ and $\varepsilon$ were assumed to be zero.

Genetic trends. Genetic trends were studied by comparing the means of the predicted breeding values of the dogs born in different years. Four breeds were investigated, namely the GS, the GR, the $\mathrm{LR}$ and the RO, as they had the largest number of observations among the breeds. The effect of timedependent effects in the model when estimating genetic trends was examined by either including (full model) or excluding (reduced model) year of birth and radiologist in the model and comparing the resulting genetic trends.

Selection differentials. Selection differentials were investigated by taking a mean value of hip and elbow dysplasia grades among the animals born in a certain year and used later in breeding, and comparing these values with the mean values of all animals born in the same year. The same four breeds were included as in the analysis of genetic trends. For hip dysplasia, years 1986 to 1997 were included, whereas for elbow dysplasia the time frame varied with breed, as the number of dogs screened was small during the late 1980s and early 1990s, varying between the breeds. The years 1991 to 1997 were included for the GS and the GR, the years 1990 to 1997 for the LR, and the years 1988 to 1997 for the RO. Obligatory hip screening for breeding dogs was established in 1984 for the Golden and the Labrador Retriever, in 1986 for the German Shepherd and in 1994 for the Rottweiler. However, using an imported dog without a screening result has been possible during the years, especially in the Labrador and the Golden Retriever. Screening for elbow dysplasia has not been mandatory until the year 2001, except for the Rottweiler, in which the official screening started in 1994. However, in the remaining three breeds, voluntary screening has been practised in elbow dysplasia for several years.

Also thresholds of the joint status for breeding dogs are different between the breeds. No thresholds exist for elbow dysplasia score in these four breeds, neither is there a threshold for the hip score of the Rottweiler. The threshold for hip dysplasia status for a German Shepherd breeding dog has been the acceptance of no worse than mildly dysplastic hips from the year 1989 onward, whereas in the Golden and the Labrador Retriever a threshold of no worse than moderate hip dysplasia has been a requirement since 1991. In the Labrador, this threshold was removed, however, at the end of the year 1999, while in the Golden Retriever, a stricter threshold, mild hip dysplasia, was adopted from the beginning of the year 2000.

Mode of inheritance. To investigate the possible existence of mitochondrial and sex-linked inheritance as well as incomplete penetrance, frequency distributions, heritabilities and regressions of offspring phenotypes on parental predicted breeding values were studied separately in males and in females. The regression approach was similar to Janss and Brascamp (1998). For the estimation of 
heritabilities, three data sets were used in each breed: the first including both sexes, the second including females and the third including males. For the regressions, the phenotypes were first regressed separately on the breeding values of sires or on the breeding values of dams. Then both the breeding values of the sire and dam were included in the model. The data were divided into two parts : only animals born until 1995 were included in the parental breeding value estimation, and only observations of the offspring born after the year 1995 were used as phenotypes. The model used in this breeding value prediction was as described above, with the exception that the $\mathrm{X}$-raying veterinarian was omitted from it, as the variance explained by it was very small according to the results of the earlier parts of this study. The regression model included the same fixed effects as the breeding value estimation model but no animal or random effects, similarly to Janss and Brascamp (1998). Expected values for these regression coefficients, when the mode of inheritance is polygenic and autosomal, are 0.5 for sires and 0.5 for dams.

Offspring frequency distributions of the males having the largest number of offspring observations on hip and elbow dysplasia were studied in order to find an indication of a segregating major gene. The number of males studied per breed was between four (RO) and twenty (GS), and the number of $X$ rayed offspring per male varied between 58 and 237 . With a major gene affecting a trait, the phenotypic frequency distribution of the progeny within a heterozygote sire would be bimodal assuming complete dominance, whereas the distribution within a homozygote sire would have only one mode - either indicating a low mean value for the offspring or showing a mean which is higher than the mean of the whole population.

Data sets and statistical programs for different analyses. Genetic trends, selection differentials, regressions and frequency distributions were each studied in the same four breeds, the GS, the GR, the LR, and the RO. Data editing and regression analyses were done with program package WSYS-L (Vilva, 1997). Variance components were estimated by Restricted Maximum Likelihood (REML) using VCE4 (Neumaier and Groeneveld, 1998). Fixed effects for the models were tested with PEST's contrast-option (Groeneveld, 1990), which performs an F-statistic by comparing the independent estimable functions of a factor. Breeding value prediction was done by best linear unbiased prediction (BLUP) using PEST.

\section{Results and discussion}

Genetic parameters

Hip dysplasia. In the data sets including both sexes, estimates of heritability for hip dysplasia varied between 0.20 (s.e. 0.03) in the $\mathrm{CO}$ and 0.41 (s.e. 0.03) in the FH (Table 1). The large heritability $\left(h^{2}\right)$ estimates in the $\mathrm{FH}$, the $\mathrm{RO}$ and the $\mathrm{BM}$ suggest that even with simple mass selection, reasonable genetic gain could be expected in these breeds. The estimates of $h^{2}$ in the GS and the RO of this study $(0.24$ (s.e. 0.02 ) and 0.37 (s.e. 0.02), respectively) were lower than in two previous studies $(0.31$ (s.e. 0.02$)$ to 0.35 (s.e. 0.02) and 0.58 (s.e. 0.04), respectively) using partly the same data but including only the dogs screened until 1996 (Leppänen et al. (2000) and Mäki et al. (2000), respectively). In the current study, the number of screened GS was 0.31 and RO 0.59 larger than in the previous studies.

In other studies, heritability of hip dysplasia has been estimated with various methods, usually by parent-offpring regression. Mostly estimates between 0.11 and 0.58 , which are of the same magnitude as in this study, have been reported (Leighton et al., 1977; Distl et al., 1991; Mäki et al., 2000; Ohlerth et al., 2001).

Litter effect accounted for 0.01 to 0.06 of the variation in hip conformation, depending on the breed (Table 1). The finding was consistent with the previous results in the same populations (Leppänen et al. (2000) and Mäki et al. (2000), respectively) as well as the results of Leighton et al. (1977) and Ohlerth et al. (2001). The litter effect includes all the non-additive genetic (dominance and epistasis) and environmental effects common to the members of the same litter. In the LR, the RO and the CO, the breeder effect was larger than the litter effect on the hip joints - the proportion of variance accounted for by the breeder varied between the breeds from 0.02 to 0.08 . However, litter and breeder effects are partly confounded.

Elbow dysplasia. For elbow dysplasia, the heritability estimates varied between $0 \cdot 10$ (s.e. 0.03) in the LR and 0.37 (s.e. 0.03) in the RO (Table 2). Compared with the previous study on Finnish Rottweilers (0.31 (s.e. 0.04); Mäki et al., 2000), the heritability estimate was a little higher in this study. In each breed, the estimates of $h^{2}$ for elbow dysplasia were lower or approximately the same as for hip dysplasia. The estimate of $h^{2}$ for the LR was surprisingly low but low estimates were obtained also for the GS and the $\mathrm{BM}$. In these breeds, a large amount of variation was accounted for by the litter effect. The number of observations on elbow dysplasia was in most breeds much smaller than on hip dysplasia, and not many dogs in a litter were screened for the trait. Thus litter 
(a)

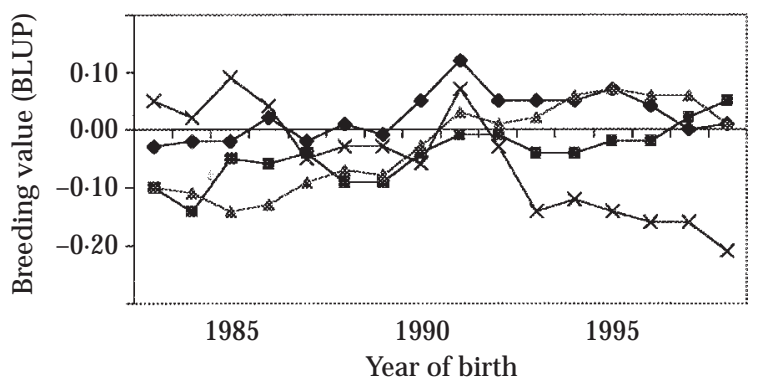

(b)

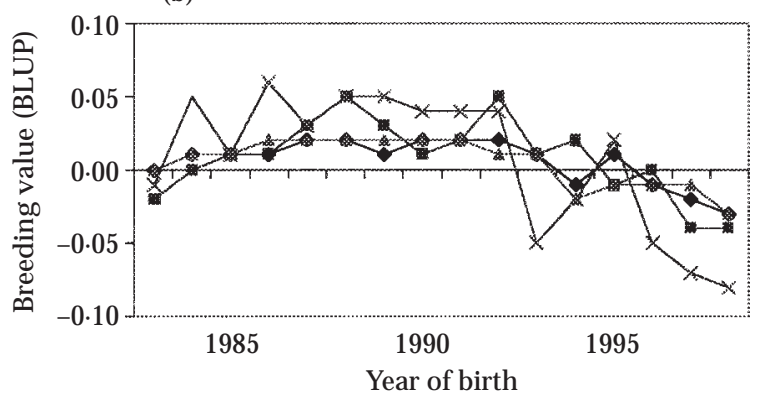

Figure 1 Genetic trends for (a) hip dysplasia and (b) elbow dysplasia $(\neg-=$ German Shepherd, $-\longrightarrow=$ Golden Retriever, $--=$ Labrador Retriever, $\ \leftarrow=$ Rottweiler).

and additive genetic effects may have been difficult to separate from each other. The effect of breeder on elbow joints was in each breed smaller than the effect of litter, except for the GS.

In other studies, estimates of heritability for elbow dysplasia or a specific form of it have mostly varied from 0.06 to 0.53 (Grøndalen and Lingaas, 1991; Janss and Brascamp, 1998; Beuing et al., 2000; Everts, 2000). All these authors except Grøndalen and Lingaas (1991) used an animal model in the estimation, as in this study.

Only a small part of the variation $(0.01$ to 0.03$)$ in both hip and elbow dysplasia was accounted for by the X-raying veterinarian. In further analyses, this effect could be left out of the models.

Genetic correlations between hip and elbow dysplasia. Estimates of genetic correlations between hip and elbow dysplasia were -0.09 in the GR, 0.00 (s.e. $0 \cdot 10$ ) in the GS, 0.19 (s.e. 0.04 ) in the RO, 0.26 (s.e. 0.10 ) in the BM and 0.31 (s.e.0.09) in the LR. In the GR, the optimization did not go through properly, and thus no standard errors were obtained. These correlations are quite low, and suggest that hip and elbow dysplasia are only partially influenced by the same or closely linked genes. Growth disorders often manifest themselves in multiple parts of a dog's skeleton. A dog with hip or elbow dysplasia may well have growth disorders in other parts of the skeleton as well. This would lead one to think that the same genes are in part involved with all the skeletal growth disorders. In the previous study on Finnish Rottweilers (Mäki et al., 2000), the genetic correlation between hip and elbow dysplasia was estimated to be 0.37 (s.e. 0.08), which is higher than the estimate obtained in this study for the same breed but not significantly different.

\section{Genetic trends and selection differentials}

Hip dysplasia. In each breed except the RO, genetic trends for hip dysplasia were unfavourable (Figure 1a). However, the changes were small, 0.04 to 0.15 hip score points in 16 years when a change of 1.00 points would indicate a transition from for example borderline to a mild dysplasia. The favourable genetic trend of the $\mathrm{RO}$ indicated an improvement of 0.26 hip score points from 1983 to 1998 .

Phenotypic selection differentials in males within a year of birth varied from 0.36 in the GS to 0.42 in the LR over the years. In females the selection differential was of the same magnitude, from 0.36 in the LR to 0.45 in the GR. Taken as a proportion of phenotypic standard deviation these figures varied between 0.28 and $0 \cdot 47$. Our data included only dogs screened in Finland, however. If available, screening results of the imported breeding dogs would probably have changed these figures. According to these estimates, hardly any selection pressure against hip dysplasia has been applied during the years studied. Because of the absence of selection, no improvement has occurred either. The small selection pressure applied may be consistent with a breeding goal to improve other characteristics while keeping the level of dysplasia constant. The RO had the highest estimate of $h^{2}$ among these four breeds, which has made the mass selection practised more effective compared with the other breeds, and resulted in a slight gain.

In 1991, a strong peak of worsened mean hip joint status could be observed among both GS and RO. It was the year during which the number of registered puppies as well as the number of imported dogs went up (Mäki et al., 2001), and it is possible that a strong demand for puppies during that time brought along a side-effect of a smaller selection intensity and a poorer health status of the breeding dogs compared with the situation before and after 1991 . The same peak, however smaller, could be seen in the other two breeds, and in the phenotypic trends in these four breeds as well, reported by Leppänen and 
Saloniemi (1999). Also, in Sweden, an increased use of foreign sires was accompanied by a slight increase in the incidence of hip dysplasia in several breeds (Hedhammar et al., 1999).

Lingaas and Klemetsdal (1990) studied the genetic trend for hip dysplasia in a Norwegian Golden Retriever population, and found a reasonable, close to proportionately 0.08 genetic improvement during the years 1975 to 1986 . This was presented as a result of a conducted phenotypic selection. The only report on the effect of BLUP-selection in hip dysplasia is by Leighton (1997). In his study on two populations of dogs for the blind, owned by The Seeing Eye Inc, the proportion of affected German Shepherds and Labrador Retrievers declined in five generations from 0.55 to 0.24 and from 0.30 to 0.10 , respectively. In the last 8 years of the study, a distraction index (DI), which is a measurement of the laxity of the hip joint, had also been used as an information source in selection besides BLUP breeding values.

Besides Lingaas and Klemetsdal (1990) and Leighton (1997), other authors have reported mainly phenotypic trends, which are not straightforward indications of genetic trends, since environmental factors often play an important rôle in the yearly changes of the mean phenotypes. Various amounts of gain have been reported in studies with various breeds (Brass, 1989; Reed et al., 2000; Wood et al., $2000 \mathrm{~b})$. However, the highest gain has been achieved mostly in closed breeding populations with systematic breeding programmes (Hedhammar et al., 1979; Leighton, 1997; Ohlerth et al., 2001). In a previous Finnish study, phenotypic progress could be detected only among Golden and Labrador Retrievers, Rottweilers and English Cockerspaniels, while in others the trends were unfavourable or nonexistent (Leppänen and Saloniemi, 1999).

Elbow dysplasia. Genetic trends for elbow dysplasia were favourable after the year 1992 in each breed : the slight improvement in the genetic health of the elbow joints varied from 0.04 to 0.12 elbow score points between the breeds (Figure 1b). Among RO the yearly fluctuations were large. Phenotypic selection differentials varied in males between $0 \cdot 13$ (the LR) and 0.17 (the RO). In females it was lower, 0.07 in the LR to $0 \cdot 10$ in the RO. As a proportion of the phenotypic standard deviations these differentials were between 0.19 and 0.52 , indicating, as with hip dysplasia, that only mild selection against elbow dysplasia has been practised. In order to lower the incidence of hip and elbow dysplasia in these breed populations, systematic selection would be needed. Large amounts of information have been gathered in the form of hip and elbow X-rays over the years. Now would be a good time finally to start using this information.

In the $\mathrm{RO}$, the overall trend for both traits between 1988 and 1995 was similar to an earlier study of Mäki et al. (2000), although the dogs born during the latter years of the earlier study now have more information for breeding value prediction. In the earlier study those dogs were too young to have offspring of their own. Additional information could also be the reason for the large changes from the year 1992 to 1993 and 1993 to 1995 observed in the current study, but not in the previous one.

In Germany, a favourable phenotypic trend for elbow dysplasia among Rottweilers was reported by Beuing et al. (2000). The proportion of unaffected dogs increased from 0.348 to 0.538 in 4 years (1996 to 1999). In Sweden and The Netherlands for example, the incidence of elbow dysplasia or specific forms of it has decreased (Swenson et al., 1997a; Ubbink et al., 1999).

Currently, estimated BLUP breeding values (EBVs) provide a more effective tool for breeding against hip and elbow dysplasia in Finnish dog breed populations. Genetic gain is expected to be larger if the breeders choose breeding dogs according to estimated breeding values instead of using only phenotypes. Until now, the poor genetic progress can be explained by the very small intensity of selection, and by the lack of EBVs.

Effect of time-dependent fixed effects on genetic trends. In both traits, differences were observed between the genetic trends estimated with the full and with the reduced model. However, for elbow dysplasia, those

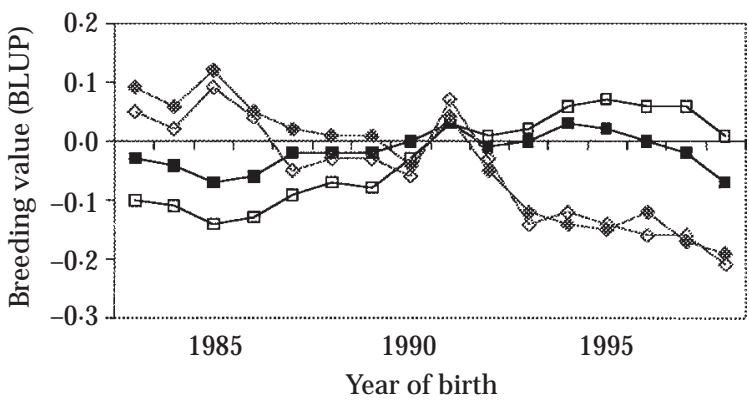

Figure 2 Effect of time-dependent fixed effects (TFE) in the model when estimating genetic trends for hip dysplasia ( - Labrador Retriever, TFE included in the model; $\square \square=$ Labrador Retriever, TFE excluded from the model; — - = Rottweiler, TFE included in the model; $\checkmark=$ Rottweiler, TFE excluded from the model). 
differences were very small except in the RO. The largest difference between the models was seen in hip dysplasia among LR (Figure 2, only two breeds and hip dysplasia showed as an example). In all four breeds except the $\mathrm{RO}$, the first years of the studied period showed worse hip joint status in the fullmodel trend than in the reduced-model trend, and starting from 1991 or 1992, it was vice versa. The yearly fluctuations in the mean breeding values for hip dysplasia and, in other breeds except in the RO, the overall change between 1983 and 1998, were all larger with the reduced model compared with the full model. This is logical as in the full model, the effect of year of birth was accounted for, and so the resulting trend showed smaller changes between the years.

Time-dependent effects are often confounded with genetic, phenotypic and/or inbreeding trends. It is generally not possible to define the 'best' model, but a sensitivity analysis to assess the effect of the model on the trend should be included to look for the robustness of the results.

\section{Mode of inheritance}

Frequency distributions of males and females. No differences were found between the frequency distributions for dysplasia scores in the two sexes. If an incomplete penetrance in either males or in females existed, either males or females would be affected more frequently compared with the other sex. Everts (2000) found males to have higher penetrance than females, based on complex segregation analysis for FCP, and males were also more frequently affected than females. Ubbink et al. (1999) studied FCP and INC in Bernese Mountain Dogs, and concluded that they were most likely genetically different traits. In the Finnish data, the four separate growth disorders of the elbow joint were not specified. They all led to the same phenotype, elbow arthrosis, according to which a dog's score for elbow dysplasia was given. This might explain the differences between the current study and the study of Everts (2000) in which only FCP was studied.

Estimates of $\mathrm{h}^{2}$ for hip dysplasia in females and in males. For hip dysplasia, differences between the estimates of $h^{2}$ in males and in females were seen in three breeds. In the GR, the CO and the BM, the estimates of $h^{2}$ were higher in males than females (Table 1). For BM males, no standard error for the heritability estimate was obtained. The standard error in males is probably of the same magnitude as in females, meaning that the estimate did not differ significantly from the estimate in females in that breed. The difference of the estimates of $h^{2}$ for hip dysplasia between males and females was largest in the CO. However, it was not statistically significant, based on 95\% confidence interval. These results are consistent with the study of Swenson et al. (1997b), in which there were no clear differences in the $h^{2}$ estimates for hip dysplasia between the sexes. Wood et al. (2000a) estimated $h^{2}$ for hip dysplasia in Gordon Setters by regression of litter mean on parent, and found no statistically significant estimate of $h^{2}$ for sires, but instead a significant estimate for dams $(0.36$ (s.e. $0 \cdot 14)$ ). For Newfoundlands, Wood et al. (2000b) reported different estimates of $h^{2}$ for sires and dams, however with large standard errors the difference was not statistically significant $(0.40$ (s.e. $0 \cdot 15)$ and 0.59 (s.e. 0.11), respectively). The findings of Wood et al. (2000a and b) were in contrast with the results of this study, as Wood et al. (2000a and b) found the estimate of $h^{2}$ to be larger in females than males.

Also the litter variance for hip dysplasia varied between males and females; however, given the standard errors, the differences were not large (Table 1 ). The division of the data into males and females affected the variance proportions, especially the proportion of litter variance, since not many screened dogs in a litter existed in the data. With litter included in the model, analysis may not have been capable of separating the additive and litter variance in this kind of a data set. Because of this, the estimates of $h^{2}$ were also calculated when excluding the litter effect from the model. Including the litter in the models did not have a large effect on the difference between males and females in terms of $h^{2}$ estimate in any of the breeds. The largest effect of the litter on this difference was seen in the RO, in which there were no difference between males and females when litter was included in the model, and a difference of 0.06 (higher in males than females) when it was excluded.

Estimates of $\mathrm{h}^{2}$ for elbow dysplasia in females and in males. For elbow dysplasia, the estimates of heritabilities differed between the two sexes in the GS and the GR (Table 2). The differences between the estimates of $h^{2}$ in males and females were strongly affected by the litter effect in the model, probably because the data sets for elbow dysplasia were fairly small in each breed. Without this effect in the model, differences between the estimates of $h^{2}$ in males and females existed also in the LR and the BM but vanished in the GS. In GS, LR and BM, the estimate of $h^{2}$ was higher in males, while among GR it was higher in females. Guthrie and Pidduck (1990) reported higher estimates of $h^{2}$ for OCD and UAP forms of elbow dysplasia in Labrador Retriever males compared with females, which is consistent with the results of this study. The standard errors in 
the study of Guthrie and Pidduck (1990) could explain the difference between the estimates for the two sexes, however.

Based on these estimates of $h^{2}$ in males and females, the pattern of inheritance of hip and elbow dysplasia seems to be polygenic and autosomal with equal parental contributions. In a few breeds the estimates differed, however not significantly, between males and females, being higher in males.

Regression analyses. For hip dysplasia, the estimated regression coefficients were similar to their expected values, taking the large standard errors into consideration (Table 3). This indicates that both parents contribute equally to their offspring in these populations: hip dysplasia seems not to be of mitochondrial or sex-linked inheritance. For elbow dysplasia, estimates of the regression coefficients were larger than their expectations. In that trait, the number of observations was much smaller than in hip dysplasia, and the calculation of the parental breeding values (EBVs) was based more on the pedigree than on actual observations. This may have affected the accuracy of the EBVs. Also the standard errors of the regression coefficients were larger in elbow than in hip dysplasia. However, in the GR dams and the LR and the GS sires, the estimates were approximately as expected; like hip dysplasia, elbow dysplasia seems to be inherited equally from the sire and from the dam. In a study of Wood et al. (2000a) on hip dysplasia of Gordon Setters, the phenotypic regressions of offspring-sire and offspring-dam were not different from each other. This is in agreement with our study, although in the study of Wood et al. (2000a), parental phenotypes were used instead of predicted breeding values. Ohlerth et al. (2001) studied the regression of offspring hip phenotypes on parental estimated breeding values, as we did, in Labrador Retrievers and came to the same conclusion with no significant deviation from the expected regression coefficient.

In a study of Janss and Brascamp (1998) on FCP in a population of Labrador Retrievers of the Royal Dutch Guide Dog for the Blind Association, the estimate of the regression coefficient of new observations on the pedigree index was -0.25 instead of the expected value 1.0. Contrary to the results of our study, Janss and Brascamp (1998) concluded that a complicated mode of inheritance, including imprinted or sex-linked genes, is likely for FCP, as the estimate of the regression coefficient of new observations on the breeding value of the dam with a maternal model was very close to its expected value. However, the number of observations in that study was very low. Everts (2000) investigated the same, only later born Labrador Retriever population as

Table 3 Estimates of regression coefficients of offspring phenotypes on their parents' predicted breeding values (EBVs); offspring born after 1995

\begin{tabular}{|c|c|c|c|c|}
\hline \multirow[b]{2}{*}{ Source of EBVs } & \multicolumn{4}{|c|}{ Breedt } \\
\hline & GS & GR & LR & $\mathrm{RO}$ \\
\hline & \multicolumn{4}{|c|}{ Hip dysplasia } \\
\hline Sire & $0 \cdot 37 \pm 0 \cdot 08$ & $0 \cdot 64 \pm 0 \cdot 13$ & $0 \cdot 33 \pm 0 \cdot 12$ & $0 \cdot 16 \pm 0 \cdot 11$ \\
\hline Dam & $0.51 \pm 0.08$ & $0 \cdot 74 \pm 0 \cdot 11$ & $0 \cdot 62 \pm 0.12$ & $0 \cdot 49 \pm 0 \cdot 10$ \\
\hline Sire + dam $\ddagger$ & $0 \cdot 36+0 \cdot 46$ & $0 \cdot 60+0 \cdot 75$ & $0 \cdot 27+0 \cdot 70$ & $0 \cdot 15+0 \cdot 45$ \\
\hline $\mathrm{N}$ sireEBV§ & 1358 & 1077 & 1020 & 693 \\
\hline N damEBV & 2093 & 1141 & 1126 & 718 \\
\hline \multirow[t]{2}{*}{$\mathrm{N}$ sire + dam } & 1258 & 1001 & 906 & 667 \\
\hline & \multicolumn{4}{|c|}{ Elbow dysplasia } \\
\hline Sire & $0 \cdot 68 \pm 0 \cdot 30$ & $1 \cdot 22 \pm 0 \cdot 20$ & $0 \cdot 44 \pm 0 \cdot 31$ & $0 \cdot 54 \pm 0 \cdot 10$ \\
\hline Dam & $0 \cdot 97 \pm 0 \cdot 27$ & $0 \cdot 48 \pm 0 \cdot 20$ & $1 \cdot 41 \pm 0 \cdot 39$ & $0 \cdot 60 \pm 0 \cdot 10$ \\
\hline Sire + dam & $0 \cdot 73+1 \cdot 16$ & $1 \cdot 19+0 \cdot 41$ & $0 \cdot 20+1 \cdot 44$ & $0 \cdot 49+0 \cdot 58$ \\
\hline N sireEBV & 1012 & 966 & 882 & 671 \\
\hline $\mathrm{N}$ damEBV & 1627 & 1022 & 974 & 695 \\
\hline $\mathrm{N}$ sire + dam & 933 & 896 & 777 & 645 \\
\hline
\end{tabular}

† GS = German Shepherd, GR = Golden Retriever, LR = Labrador Retriever, RO = Rottweiler.

$\ddagger$ For example $0.36+0.46=0.36$ for the sire and 0.46 for the dam.

$\S \mathrm{N}$ sireEBV $=$ number of offspring phenotypes in the regression analysis on sire's EBV. 
Janss and Brascamp (1998), and reported that, based on a complex segregation analysis, a major gene model best described FCP.

Frequency distributions of the progeny phenotypes within individual sires. Offspring distributions were nearly normal for males with most offspring classified as mildly dysplastic, and positively skewed (the tail to the right was longer than that to the left) in those whose offspring were mainly of normal or borderline phenotype. No sign of bimodality or negative skewness was noticed within any of the sires. This does not, however, rule out the feasibility of a major gene in either of the traits. A segregation analysis would be needed in order to assess segregation of such genes with a large effect.

For hip dysplasia, the possibility of an existing major gene in populations of German Shepherd Dog and Labrador Retriever has been reported by Leighton (1997), who calculated a major gene index (MGI) according to LeRoy and Elsen (1992). Based on linkage analysis in a population of Labrador Retriever $\times$ Greyhound dogs, Todhunter et al. (1999) suggested that at least two major genes are influencing hip dysplasia in dogs. Everts (2000) searched possible markers for FCP, but concluded that approximately $80 \%$ of the dog genome was excluded as a candidate region, under a hypothesis of a recessive inheritance. Involvement of the $X$ chromosome could not be substantiated either. Moreover, even if each of the disorders of the elbow joint were caused by one or two single genes, the overall elbow arthrosis, used in Finland in grading the elbow joints, would be a trait with a polygenic mode of inheritance.

\section{Conclusions}

A large amount of additive genetic variation existed in hip and elbow joint conformation in most of the dog breeds in this study. The estimates of $h^{2}$ were lower for elbow dysplasia than for hip dysplasia, especially in the LR, the GS and the BM.

Genetic trends for hip dysplasia between the years 1983 and 1998 were favourable only in the RO. In elbow dysplasia, the trends showed a slight improvement after the year 1992 in all the four breeds studied. Based on phenotypic estimates, the reason for the poor progress seemed to be the small selection differentials. In order to lower the incidence of hip and elbow dysplasia in these breed populations, systematic selection would be needed. Time-dependent fixed effects, i.e. year of birth and the radiologist, did have an influence on the estimated genetic trends, resulting either in a more negative or more positive genetic trend compared with the model from which time-dependent effects were removed. It is generally not possible to define the 'best' model, but a sensitivity analysis to assess the effect of the model on the trend should be included to look for the robustness of the results.

The regression coefficients of the offspring phenotypes on parental breeding values were approximately equal to their expectations, i.e. $0 \cdot 5$, and the frequency distributions of the traits were similar among males and females. Heritability estimates of different size in males compared with females were found for hip dysplasia in the $\mathrm{CO}$ and for elbow dysplasia in the GS and the GR. However, the differences were not statistically significant. Moreover, the data sets for elbow dysplasia were small, and also the litter effect had an effect on the magnitude of the difference of the $h^{2}$ estimates for elbow dysplasia between the two sexes. No sign of single genes with a large effect affecting the traits was found in the frequency distributions of the progeny within individual sires, although this does not rule out the possible existence of a major gene. These results show that neither hip nor elbow dysplasia seem to be sex-linked or mitochondrially inherited traits in the populations studied, and that, according to this study, a quantitative or mixed mode of inheritance is the most probable.

\section{Acknowledgements}

Data for this study were provided by the Finnish Kennel Club. The study was financed, in part, by the Science Foundation of the Helsinki University, the Finnish Kennel Club, and the breed associations of the Finnish Hound, the Golden Retriever and the Rottweiler. The authors want to thank Luc Janss, Anna Sonesson, Piter Bijma and the two anonymous referees for their useful suggestions for this study.

\section{References}

Beuing, R., Mues, Ch., Tellhelm, B. and Erhardt, G. 2000. Prevalence and inheritance of canine elbow dysplasia in German Rottweiler. Journal of Animal Breeding and Genetics 117: 375-383.

Brass, W. 1989. Hip dysplasia in dogs. Journal of Small Animal Practice 30: 166-170.

Distl, O., Grussler, W., Schwarz, J. and Kräusslich, H. 1991. [Analysis of environmental and genetic influences on the frequency of hip dysplasia in German Shepherd dogs. ] Journal of Veterinary Medicine 38: 460-471.

Everts, R. E. 2000. Molecular genetic studies in the dog; application to fragmented coronoid process (FCP) in the Labrador Retriever. Ph. D. thesis, Faculty of Veterinary Medicine, Utrecht University, The Netherlands.

Groeneveld, E. 1990. PEST user's manual. Institute of Animal Husbandry and Animal Behaviour, Federal Agricultural Research Centre, Germany. 
Grøndalen, J. and Lingaas, F. 1991. Arthrosis in the elbow joint of young rapidly growing dogs: a genetic investigation. Journal of Small Animal Practice 32: 460-464.

Guthrie, S. and Pidduck, H. G. 1990. Heritability of elbow osteochondrosis within a closed population of dogs. Journal of Small Animal Practice 31: 93-96.

Hedhammar, Å., Olsson, S. -E., Andersson, S. -Å., Persson, L., Pettersson, L., Olausson, A. and Sundgren, P. -E. 1979. Canine hip dysplasia: study of heritability in 401 litters of German Shepherd dogs. Journal of the American Veterinary Medical Association 174: 1012-1064.

Hedhammar, Å., Swenson, L. and Egenvall, A. 1999. Elbow arthrosis and hip dysplasia in Swedish dogs as reflected by screening programmes and insurance data. European Journal of Companion Animal Practice 9: 119-121.

Janss, L. L. G. and Brascamp, E. W. 1998. Prediction of elbow dysplasia in dogs by breeding values of relatives. Proceedings of the sixth world congress of genetics applied to livestock production, Armidale, vol. 25, pp. 621-624.

Leighton, E. A. 1997. Genetics of canine hip dysplasia. Journal of the American Veterinary Medical Association 210: 1474-1479.

Leighton, E. A., Linn, J. M., Willham, R. L. and Castleberry, M. W. 1977. A genetic study of canine hip dysplasia. American Journal of Veterinary Research 38: 241-244.

Leppänen, M., Mäki, K., Juga, J. and Saloniemi, H. 2000. Estimation of heritability for hip dysplasia in German Shepherd dogs in Finland. Journal of Animal Breeding and Genetics 117: 97-103.

Leppänen, M. and Saloniemi, H. 1999. Screening and controlling canine hip dysplasia in Finland. Preventive Veterinary Medicine 42: 121-131.

LeRoy, P. and Elsen, J. M. 1992. Simple test statistics for major gene detection: a numerical comparison. Theoretical and Applied Genetics 83: 635-644.

Lingaas, F. and Klemetsdal, G. 1990. Breeding values and genetic trend for hip dysplasia in the Norwegian Golden Retriever population. Journal of Animal Breeding and Genetics 107: 437-443.

Mäki, K., Groen, A. F., Liinamo, A. -E. and Ojala, M. 2001. Population structure, inbreeding trend and their association with hip and elbow dysplasia in dogs. Animal Science 73: 217-228.

Mäki, K., Liinamo, A.-E. and Ojala, M. 2000. Estimates of genetic parameters for hip and elbow dysplasia in Finnish Rottweilers. Journal of Animal Science 78: 1141-1148.
Neumaier, A. and Groeneveld, E. 1998. Restricted maximum likelihood estimation of covariances in sparse linear models. Genetics, Selection, Evolution 30: 3-26.

Ohlerth, S., Lang, J., Busato, A. and Gaillard, G. 2001. Estimation of genetic population variables for six radiographic criteria of hip dysplasia in a colony of Labrador Retrievers. American Journal of Veterinary Research 62: $846-852$.

Reed, A. L., Keller, G. G., Vogt, D. W., Ellersieck, M. R. and Corley, E. A. 2000. Effect of dam and sire qualitative hip conformation scores on progeny hip conformation. Journal of the American Veterinary Medical Association 217: 675-680.

Swenson, L., Audell, L. and Hedhammar, Å. 1997a. Prevalence and inheritance of and selection for elbow arthrosis in Bernese Mountain Dogs and Rottweilers in Sweden and benefit: cost analysis of a screening and control program. Journal of the American Veterinary Medical Association 210: 215-221.

Swenson, L., Audell, L. and Hedhammar, Å. 1997b. Prevalence and inheritance of and selection for hip dysplasia in seven breeds of dogs in Sweden and benefit: cost analysis of a screening and control program. Journal of the American Veterinary Medical Association 210: 207-214.

Todhunter, R. J., Acland, G. M., Olivier, M., Williams, A. J., Vernier-Singer, M., Burton-Wurster, N., Farese, J. P., Grohn, Y. T., Gilbert, R. O., Dykes, N. L. and Lust, G. 1999. An outcrossed canine pedigree for linkage analysis of hip dysplasia. The Journal of Heredity 90: 83-92.

Ubbink, G. J., Hazewinkel, H. A. W., Broek, J. van de and Rothuizen, J. 1999. Familial clustering and risk analysis for fragmented coronoid process and elbow joint incongruity in Bernese Mountain Dogs in The Netherlands. American Journal of Veterinary Research 60: 1082-1087.

Vilva, V. 1997. Data preparation system WSYS-L. http: // www. animal. helsinki. fi/wsysl/wsysl. html.

Wood, J. L. N., Lakhani, K. H. and Dennis, R. 2000a. Heritability of canine hip-dysplasia score, and its components in Gordon Setters. Preventive Veterinary Medicine 46: 87-97.

Wood, J. L. N., Lakhani, K. H. and Dennis, R. 2000 b. Heritability and epidemiology of canine hip-dysplasia score in Flat-Coated Retrievers and Newfoundlands in the United Kingdom. Preventive Veterinary Medicine 46: 75-86.

(Received 21 November 2001-Accepted 29 April 2002) 